\title{
BMJ Open Impact of valve morphology, hypertension and age on aortic wall properties in patients with coarctation: a two-centre cross-sectional study
}

Niky Ghorbani, ${ }^{1,2}$ Vivek Muthurangu, ${ }^{3}$ Abbas Khushnood, ${ }^{3}$ Leonid Goubergrits, ${ }^{1,2}$ Sarah Nordmeyer, ${ }^{1,2}$ Joao Filipe Fernandes, ${ }^{1}$ Chong-Bin Lee, ${ }^{1,4}$ Kilian Runte, ${ }^{1}$ Sophie Roth, ${ }^{1}$ Stephan Schubert, ${ }^{2,5}$ Sebastian Kelle, ${ }^{4,5}$ Felix Berger, ${ }^{2,5}$ Titus Kuehne, ${ }^{1,2,5}$ Marcus Kelm (1) 1,2,6

To cite: Ghorbani N, Muthurangu V, Khushnood A, et al. Impact of valve morphology, hypertension and age on aortic wall properties in patients with coarctation: a two-centre crosssectional study. BMJ Open 2020;10:e034853. doi:10.1136/ bmjopen-2019-034853

- Prepublication history for this paper is available online. To view these files, please visit the journal online (http://dx.doi. org/10.1136/bmjopen-2019034853).

Received 18 November 2019 Revised 13 February 2020 Accepted 04 March 2020

Check for updates

(C) Author(s) (or their employer(s)) 2020. Re-use permitted under CC BY-NC. No commercial re-use. See rights and permissions. Published by BMJ.

For numbered affiliations see end of article.

Correspondence to

Dr Marcus Kelm;

mkelm@dhzb.de

\section{ABSTRACT}

Objective We aimed to investigate the combined effects of arterial hypertension, bicuspid aortic valve disease (BAVD) and age on the distensibility of the ascending and descending aortas in patients with aortic coarctation. Design Cross-sectional study.

Setting The study was conducted at two university medical centres, located in Berlin and London.

Participants A total of 121 patients with aortic coarctation (ages 1-71 years) underwent cardiac MRI, echocardiography and blood pressure measurements. Outcome measures Cross-sectional diameters of the ascending and descending aortas were assessed to compute aortic area distensibility. Findings were compared with age-specific reference values. The study complied with the Strengthening the Reporting of Observational Studies in Epidemiology statement and reporting guidelines.

Results Impaired distensibility (below fifth percentile) was seen in $37 \%$ of all patients with coarctation in the ascending aorta and in $43 \%$ in the descending aorta. BAVD $(43 \%)$ and arterial hypertension (72\%) were present across all ages. In patients $>10$ years distensibility impairment of the ascending aorta was predominantly associated with BAVD (OR 3.1, 95\% Cl 1.33 to $7.22, p=0.009$ ). Distensibility impairment of the descending aorta was predominantly associated with arterial hypertension (0R 2.8, 95\% $\mathrm{Cl} 1.08$ to $7.2, p=0.033$ ) and was most pronounced in patients with uncontrolled hypertension despite antihypertensive treatment.

Conclusion From early adolescence on, both arterial hypertension and BAVD have a major impact on aortic distensibility. Their specific effects differ in strength and localisation (descending vs ascending aorta). Moreover, adequate blood pressure control is associated with improved distensibility. These findings could contribute to the understanding of cardiovascular complications and the management of patients with aortic coarctation.

\section{INTRODUCTION}

Aortic coarctation (CoA) accounts for $5 \%-10 \%$ of all congenital heart defects. ${ }^{1}$ Despite progress made in early treatment

\section{Strengths and limitations of this study}

- The two-centre study investigates the combined impact of arterial hypertension, bicuspid aortic valve disease and age on the presence, location and severity of aortic distensibility impairment in patients with aortic coarctation.

- The study further characterises the effects of antihypertensive treatment and adequate blood pressure control on aortic distensibility.

- Using observational cross-sectional data precludes the authors from directly inferring causal relationships and individual longitudinal courses of distensibility alterations.

concepts, morbidity and mortality remain high. $^{2}$ Arterial hypertension (HTN) persists in more than $65 \%$ of all patients despite successful repair, ${ }^{13}$ commonly leading to cardiovascular and cerebrovascular complications and subsequently impaired long-term outcome. ${ }^{23}$ In up to $75 \%$ of cases ${ }^{4}$ CoA occurs in combination with bicuspid aortic valve disease (BAVD), which is also associated with vascular complications such as dilation of the aorta.

HTN, BAVD and age are each known to affect the distensibility of the aorta and thus on its Windkessel function that usually enables partial storage of left ventricular stroke volume during systole and the maintenance of continuous organ perfusion during diastole. Persisting HTN has been demonstrated to be associated with impaired elastic capacity of the aorta ${ }^{5}$ and thus an increased afterload that can contribute to relevant cardiovascular remodelling processes. In addition to HTN, the elasticity of the ascending aorta is frequently impaired in BAVD, as wall stresses ${ }^{6}$ are typically elevated and elastic fibre content 
of the medial layer decreases ${ }^{7}$ in the presence of permanent non-laminar flow profiles. Furthermore, ageing has been shown to contribute to a loss of vascular elasticity throughout life. ${ }^{8}$

Although HTN, BAVD and age have each been associated with impaired elasticity, little is known about their combined impact. The specific impact of each risk factor on the impairment of vascular properties and the effects of antihypertensive treatment can be of clinical relevance. We therefore aimed to investigate the combined effects of HTN (with and without antihypertensive treatment), BAVD and age on the presence, location and severity of aortic distensibility impairment in patients with CoA using a non-invasive MRI-based approach.

\section{METHODS}

\section{Study design, population and patient involvement}

This observational study was carried out at two centres, located in Berlin, German Heart Centre Berlin $(n=84)$ and London, University College London $(n=37)$. The study population consisted of 121 consecutive outpatients with known CoA between January 2014 and December 2016. Key inclusion criteria for the MRI study were (1) a confirmed diagnosis of CoA with (2) an indication for diagnostic evaluation according to European Society of Cardiology (ESC) $)^{9}$ and American College of Cardiology/American Heart Association (ACC/AHA) guidelines $^{10}$ due to (2A) echocardiography or blood pressure measurements indicating pressure gradients across the stenosis exceeding $20 \mathrm{~mm} \mathrm{Hg}$ or (2B) severe narrowing and/or (2C) HTN that were (3) without relevant contraindications for MRI and were (4) without any additional complex congenital cardiovascular malformations. As this was a non-invasive study, MRI was also performed in patients with unclear treatment indications, where ESC and/or ACC/AHA guideline indications where suspected. The study complied with the Strengthening the Reporting of Observational Studies in Epidemiology statement.

In this study, all patients underwent echocardiography and cardiac MRI in conjunction with blood pressure measurements of the upper extremities. MRI-based approaches have recently been demonstrated to be feasible and reliable measures for non-invasive assessment of aortic compliance and the resulting distensibility in patients with CoA. ${ }^{11}{ }^{12}$ Patients were considered hypertensive (HTN group) where the diagnosis of HTN was made according to clinical guidelines ${ }^{13} 14$ and with consideration of paediatric percentiles where appropriate. ${ }^{14}$ For all computations, blood pressures at the time of MRI examination in comfortably placed patients with back support for at least 5 min were used. Baseline characteristics are shown in table 1.

The diagnosis of BAVD was made based on cine MRI data, acquired orthogonally to the native valve plane. Calculated aortic area distensibility was compared with percentiles of healthy individuals published by Voges $e t$ $a l .{ }^{15}$ Distensibility impairment was defined as patients with measurements below the fifth percentile.

\section{Patient and public involvement}

We did not directly include patient and public involvement in this study, but the research design has been motivated by direct interactions and regular discussions with our patients. We are also sharing study results with participants and provide a plain-language summary for patients and patient and public involvement representatives on request.

\section{Image acquisition}

Data were acquired on $1.5 \mathrm{~T}$ clinical MR systems (including Achieva; Philips Healthcare, Best, The Netherlands; and Avanto, Siemens, Erlangen, Germany). The cardiac MRI protocol included standard balanced fast field echo cine imaging with at least two slices covering the ascending and descending aortas to assess diameter changes for each vessel. The imaging planes were positioned perpendicular to the vessel at the level of the pulmonary artery bifurcation. In case of imaging artefacts due to previous stenting, the plane covering the descending aorta was placed more distally, yet remained above the level of the diaphragm. Typical imaging parameters were: voxel size $1.80 \times 1.70 \times 6 \mathrm{~mm}$, reconstructed voxel size $1 \times 1 \times 6 \mathrm{~mm}$, echo time $=1.2 \mathrm{~ms}$, repetition time $=2.5 \mathrm{~ms}$, flip angle $60^{\circ}$, retrospective cardiac gating, 40 automatically reconstructed cardiac phases. Scan duration in total was 9-14 min.

\section{Image postprocessing and analysis}

MRI cine images were analysed manually using View Forum (Philips Medical Systems Nederland; View Forum R6.3V1L7 SP1). Analysis was carried on-site for the respective patient group. The slices to be examined were manually selected at the level of pulmonary artery bifurcation (figure 1). Cross-sectional diameters of the ascending and descending aortas were measured during the end-systolic and end-diastolic heart phase, during the maximal and minimal expansion of the vessel. For each localisation, three slices nearby the point of maximal/minimal visual vessel extension were selected. For each measurement, three diameters were measured and the average was calculated. Diameters were chosen at the shortest distance and were subsequently converted to cross-sectional areas in order to minimise minor angulation errors. The arterial compliance is defined as the change in arterial blood volume $(\Delta \mathrm{V})$ relative to a given change in the arterial blood pressure $(\Delta \mathrm{P}): \mathrm{C}=\Delta \mathrm{V} / \Delta \mathrm{P}$. Clinically, an alternative version of arterial compliance is used in which the crosssectional area (A) of the aorta replaces the vessel volume. Aortic area compliance in this paper is defined as the change in the cross-sectional area of the aorta per unit of change in pulse pressure (PP):

$$
C_{\text {area }}=\frac{A_{\max }-A_{\min }}{P P_{\text {aorta }}}
$$

To achieve comparability within the study population, cross-sectional aortic area compliance was converted to 
Table 1 Patient characteristics; median and lower and upper quantiles (Q1;Q3) and n (\%)

\begin{tabular}{|c|c|c|c|c|}
\hline & $\begin{array}{l}\text { Total study group } \\
(n=121)\end{array}$ & $\begin{array}{l}\text { Non-HTN group } \\
(\mathrm{n}=34)\end{array}$ & $\begin{array}{l}\text { HTN group } \\
(n=87)\end{array}$ & $P$ value \\
\hline \multicolumn{5}{|l|}{ Characteristics } \\
\hline Male $(n, \%)$ & $80(66)$ & $22(65)$ & $58(67)$ & 0.838 \\
\hline Weight (kg) & $66.2(51 ; 78)$ & $59.25(50.2 ; 74)$ & $68(51 ; 80)$ & 0.183 \\
\hline $\mathrm{BSA}\left(\mathrm{m}^{2}\right)$ & $1.74(1.5 ; 1.95)$ & $1.66(1.47 ; 1.93)$ & $1.8(1.47 ; 1.97)$ & 0.190 \\
\hline Bicuspid aortic valve (n, \%) & $52 / 121(43)$ & $15 / 34(44)$ & $37 / 87(43)$ & 0.874 \\
\hline Previous episodes of HTN and/or exercise HTN (n, \%) & $98 / 121(81)$ & $11 / 34(32)$ & $87 / 87(100)$ & 0.002 \\
\hline Systolic BP (mm Hg) & $136(122 ; 148)$ & $116(110 ; 122)$ & $142(134 ; 152)$ & $<0.001$ \\
\hline $\begin{array}{l}\text { Patients with echocardiographic pressure drop above } \\
20 \mathrm{~mm} \mathrm{Hg} \mathrm{(n,} \mathrm{\% )}\end{array}$ & $81(67)$ & $23(68)$ & $58(67)$ & 0.918 \\
\hline \multicolumn{5}{|l|}{ Previous treatment } \\
\hline Balloon angioplasty (n, \%) & $45 / 121(37)$ & 9/34 (26) & $36 / 87(41)$ & 0.129 \\
\hline Stenting $(n, \%)$ & 23/121 (19) & $4 / 34(12)$ & $19 / 87(22)$ & 0.206 \\
\hline Surgical reconstruction (n, \%) & $73 / 121(60)$ & $23 / 34(68)$ & $50 / 87(57)$ & 0.306 \\
\hline Antihypertensive medication (n, \%) & $37 / 121(31)$ & $11 / 34(32)$ & $26 / 87(30)$ & 0.791 \\
\hline \multicolumn{5}{|l|}{ Type of medication } \\
\hline Beta blockers (n, \%) & 23/121 (19) & $7 / 34(21)$ & $16 / 87(18)$ & 0.783 \\
\hline Ascending aorta, maximum (mm) & $29.09(24 ; 33.1)$ & $29.45(24 ; 32.7)$ & $28.4(23.8 ; 33.8)$ & 0.762 \\
\hline Descending aorta, minimum (mm) & $17.9(14.7 ; 20.9)$ & $16.84(13.8 ; 20.3)$ & $17.9(15 ; 21)$ & 0.378 \\
\hline Descending aorta, maximum (mm) & $20(16.2 ; 23.3)$ & $19.25(15.8 ; 23)$ & $20.1(16.2 ; 23.4)$ & 0.500 \\
\hline
\end{tabular}

Pressure drop across the stenosis: maximal pressure in $\mathrm{mm} \mathrm{Hg}$ across the coarctated segment measured by echocardiography; ascending and descending aortas (minimum) describing end-diastolic cross-sectional diameter; ascending and descending aortas (maximum) describing end-systolic cross-sectional diameter.

Continuous data are expressed as median and IQR (Q1;Q3), p values are from Wilcoxon-Mann-Whitney test. Categorical data are presented as frequencies and percentage (\%), $\mathrm{p}$ values are from Pearson's $\chi^{2}$ test.

BMI, body mass index; BP, blood pressure; BSA, body surface area; HTN, hypertension.

area distensibility $\left(\mathrm{D}_{\text {area }}\right)$, defined as the relative change in the cross-sectional area of the aorta.

$$
D_{\text {area }}\left(\frac{1}{m m H g \times 10^{3}}\right)=\frac{A_{\max }-A_{\min }}{A_{\min }} \times \frac{1}{P P}
$$

$A_{\max }$ represents the systolic cross-sectional area and $\mathrm{A}_{\min }$ the diastolic area. PP is defined as the difference between systolic and diastolic pressure $\left(\mathrm{P}_{\text {svstolic }}-\mathrm{P}_{\text {diastolic }}\right)$. Previous studies have demonstrated ${ }^{12}$ that a non-invasive sphygmomanometer-based blood pressure measurement on the left arm can be expected to be higher than aortic PP. Hence, we used a linear model for generating the aortic PP with given values such as mean arterial pressure (MAP) $\left(M A P=\frac{2}{3} x P_{\text {diastolic }}+\frac{1}{3} x P_{\text {systolic }}\right)$ and PP measured by cuff on the left arm $\left(\mathrm{PP}_{\mathrm{LA}}\right)$, as described in Kelm et $a l^{12}$ :

$$
P P_{\text {aorta }}=P P_{L A} x\left(0.3133 x M A P_{L A}+28.366\right) /\left(M A P_{L A}-22\right)
$$

The linear model is only valid on the condition that the aortic PP is less than or equal to peripheral PP.

\section{Statistical analysis}

Continuous data are expressed as median and IQR (Q1;Q3) unless stated otherwise. Categorical data are 


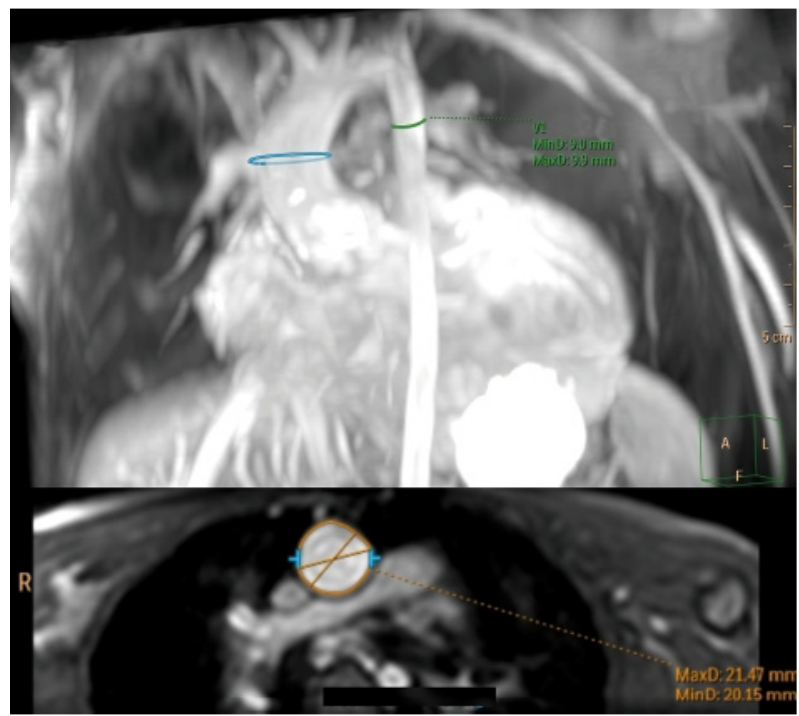

Figure 1 Locations of cross-sectional diameter measurements of the ascending and descending aortas, measured during the end-systolic and end-diastolic heart phase

presented as frequencies and percentages (\%). Data distribution was tested using Shapiro-Wilk and ShapiroFrancia tests. Wilcoxon-Mann-Whitney test was used to assess continuous data for differences between groups (HTN vs non-HTN). Pearson's $\chi^{2}$ test was used in conjunction with Fisher's exact test to compare categorical variables between groups. Non-parametric regression was performed to assess multifactorial effects on the distensibility of the aorta. Multifactorial influences leading to distensibility impairment were assessed using logistic regression. Predictive margins were calculated and plotted to visualise the combined effects of BAVD, HTN, medication and age. Stata V.15.1 was used for statistical analysis. $\mathrm{P}$ values $<0.05$ were considered statistically significant.

\section{RESULTS}

A two-centre cohort of patients with CoA was included in the analysis, in which the patient populations did not significantly differ between the participating centres. Seventy-two per cent $(87 / 121)$ of patients were hypertensive at the time of examination. BAVD was found in $43 \%$ $(52 / 121)$ of subjects. Patient characteristics of the study population divided into the two groups (HTN group and non-HTN group) are shown in table 1 . There were no significant differences between the two groups regarding patients' characteristics or previous treatment. Median echocardiographic pressure gradients and the number of patients with a pressure drop above $20 \mathrm{~mm} \mathrm{Hg}$ did not differ between groups.

In patients above the age of 10 years, aortic distensibility of the ascending and descending aortas decreased with age (figure 2). While no significant differences in distensibility of the ascending aorta were found between normotensive patients and patients with HTN (figure 2A), a multifactorial model revealed that patients with HTN and CoA above the age of 10 years showed significantly lower distensibility of the descending aorta compared with normotensive subjects in their age groups (figure 2B). In patients with CoA and coexisting BAVD older than 10 years of age, distensibility of the ascending aorta was significantly lower than patients with tricuspid aortic valve (figure 2C), while BAVD showed no impact on the distensibility of the descending aorta (figure 2D). These effects were not found in patients below the age of 10 years.

Of the total cohort, 37 patients were under antihypertensive medication. From these patients, 19 were on monotherapy and 18 were on dual or triple therapy. Compared with normotensive patients without antihypertensive medication, the estimated distensibility of the descending aorta was (1) significantly lower in normotensive patients, who were under antihypertensive treatment at the time of examination (estimated effect: $-1.32 \times 10^{-3} \mathrm{~mm} \mathrm{Hg}^{-1}, \mathrm{p}=0.031$ ). Furthermore (2), in patients with HTN without antihypertensive medication even further reduced distensibility of the descending aorta (estimated effect: $-2.43 \times 10^{-3} \mathrm{~mm} \mathrm{Hg}^{-1}, \mathrm{p}=0.020$ ) was found, and (3) was lowest in patients with HTN and CoA under antihypertensive medication, that is, patients with uncontrolled HTN despite therapy (estimated effect: $\left.-3.40 \times 10^{-3} \mathrm{~mm} \mathrm{Hg}^{-1}, \mathrm{p}=0.022\right)$. There were no drug-specific effects on distensibility of the ascending or descending aorta. The effects are illustrated in figure 3. No significant differences were found between previous surgical or interventional treatment.

In comparison to reference data from healthy individuals, ${ }^{15}$ distensibility impairment below the fifth percentile was found in the ascending aorta in $37.2 \%$ and in the descending aorta in $43.0 \%$ of all patients with CoA. Patients with CoA with a combination of BAVD and HTN had impaired distensibility of the ascending aorta in $49.6 \%$ and distensibility impairment of the descending aorta in $51.4 \%$. In normotensive patients with CoA and physiological tricuspid valve, impaired distensibility was present in just $21.1 \%$ in the ascending aorta and $26.3 \%$ in the descending aorta.

In patients below 10 years of age, no associations were found between BAVD and impaired distensibility in the ascending $(\mathrm{p}=0.519)$ or the descending $(\mathrm{p}=0.889)$ aorta. Also, HTN and impaired distensibility showed no significant correlation, either in the ascending $(\mathrm{p}=0.635)$ or in the descending $(\mathrm{p}=0.207)$ aorta. In patients above the age of 10 years, distensibility impairment of the ascending aorta was more common in BAVD (OR 3.1, 95\% CI 1.33 to $7.22, p=0.009$ ). In these patients with BAVD, impaired distensibility of the ascending aorta was seen in $51.1 \%$, compared with $26.9 \%$ in patients with a tricuspid valve. Associations between BAVD and impaired distensibility of the ascending aorta further increased with age, whereas no such associations were found in the descending aorta. Patients with HTN above the age of 10 years showed no association with distensibility impairment in the ascending 

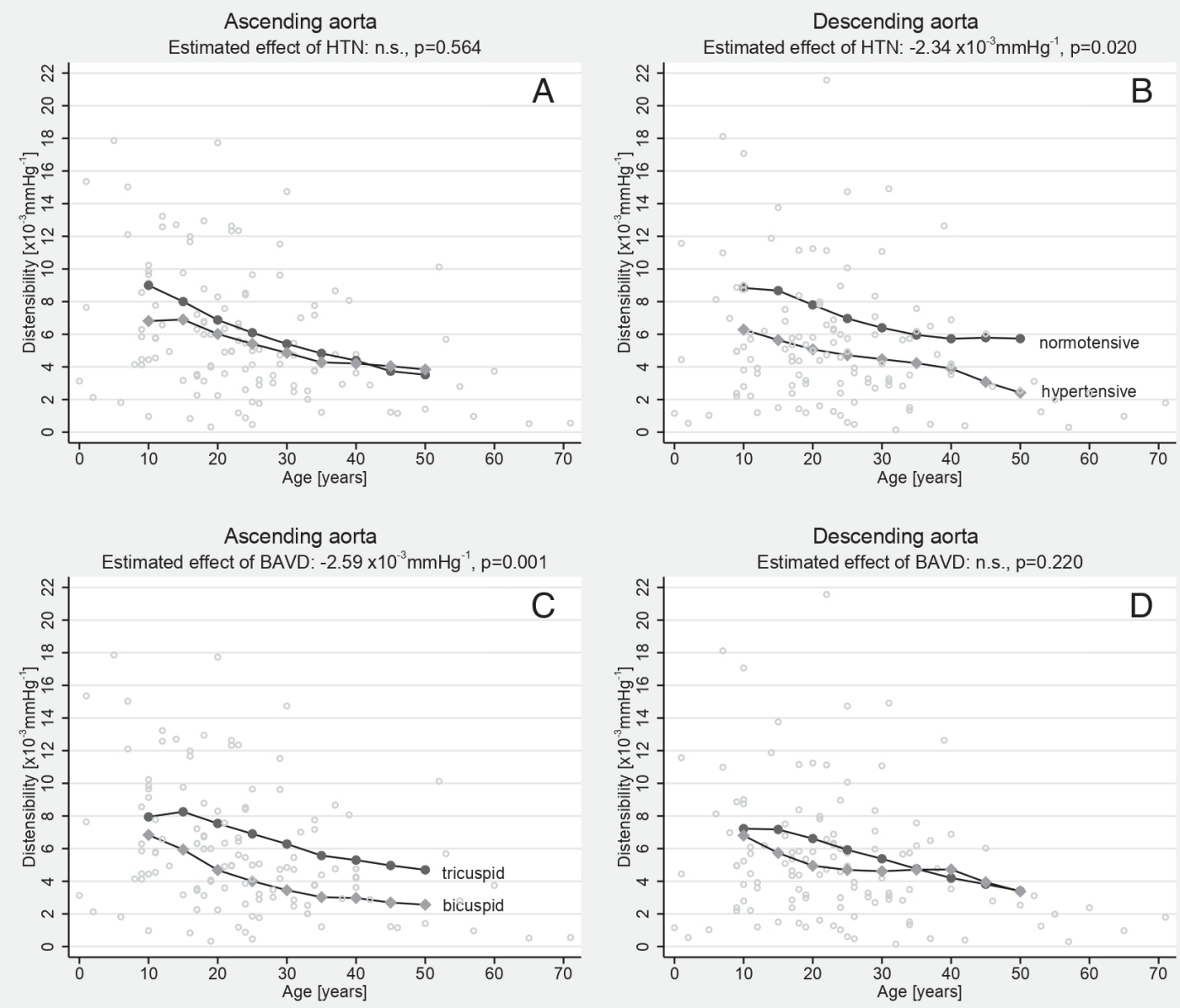

Figure 2 Aortic distensibility of the ascending aorta (left) and descending aorta (right) in patients with or without accompanying HTN or BAVD, plotted against age. It includes the effects of (A) HTN on the ascending aorta, (B) HTN on the descending aorta, (C) BAVD on the ascending aorta and (D) BAVD on the descending aorta; BAVD, bicuspid aortic valve disease; HTN, hypertension.

aorta $(\mathrm{p}=0.239)$, whereas distensibility impairment of the descending aorta was correlated with HTN (OR 2.8, $95 \%$ CI 1.08 to $7.2, p=0.033)$. Distensibility impairment of

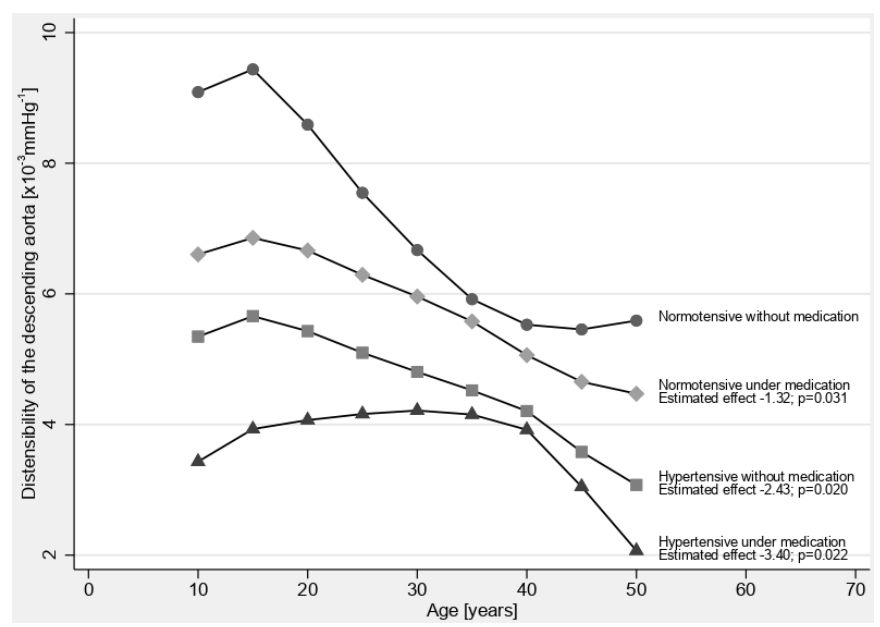

Figure 3 Estimated effects of antihypertensive medication on the distensibility of the descending aorta, compared with normotensive patients without medication (reference). the descending aorta below the fifth percentile was seen in $49.3 \%$ of all patients with HTN $>10$ years, compared with $26.7 \%$ in normotensive patients.

The combined impact of BAVD and HTN on distensibility impairment below the fifth percentile in patients with CoA aged 10-50 years is shown in figure 4 . In patients with HTN, 95\% CIs of the estimated risk for distensibility impairment of the descending aorta did not overlap from the age of 30 years onwards. In patients with BAVD, the same effects were observed for the ascending aorta in patients above 30 years.

Significant differences in cross-sectional diameters of the ascending aorta between patients with BAVD and patients with tricuspid valve and CoA were observed. Compared with patients with tricuspid aortic valve, those with BAVD had significantly larger end-diastolic and end-systolic diameters of the ascending aorta: minimum $22.6(18.9 ; 28.5) \mathrm{mm}$ in tricuspid valve morphology vs 28 $(22.3 ; 36.1) \mathrm{mm}$ in BAVD, $\mathrm{p}<0.001$; maximum 27 (21.9;31) $\mathrm{mm}$ vs $30.7(24.9 ; 38.7) \mathrm{mm}$ in BAVD, $\mathrm{p}=0.002$. 

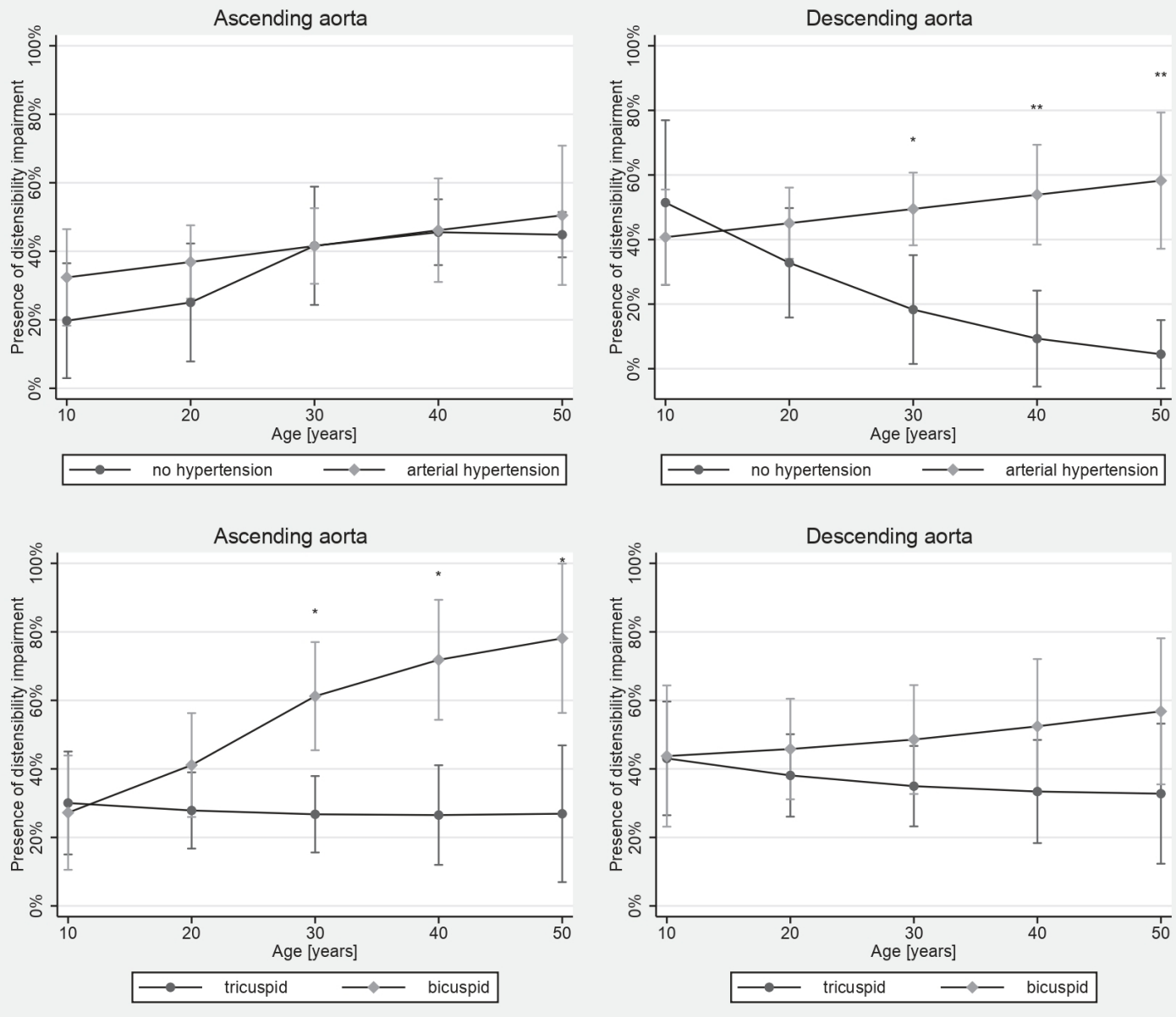

Figure 4 Marginal effects and 95\% Cls for impaired aortic distensibility (below the fifth percentile) of the ascending and descending aortas, in patients with or without hypertension (HTN) or bicuspid aortic valve disease (BAVD), plotted against age $\left({ }^{*} \mathrm{p}<0.05 ;{ }^{* *} \mathrm{p}<0.01\right)$.

\section{DISCUSSION}

In our cohort, aortic distensibility was impaired in almost half of all patients with CoA. In those above the age of 10 years, BAVD had a significant effect on distensibility impairment of the ascending aorta, whereas HTN predominantly affected the descending aorta. Additionally, antihypertensive treatment was associated with improved distensibility. However, when compared with normotensive patients without medical treatment, normotensive patients under antihypertensive medication had significantly lower distensibility across all ages. Distensibility was even lower in untreated patients with HTN, and lowest in those with uncontrolled HTN despite antihypertensive therapy.

Increased aortic stiffness (impairment of the Windkessel capacity) is considered as a marker for vascular disease and cardiovascular outcome. ${ }^{16}$ An example can demonstrate the immediate impact of reduced distensibility on Windkessel capacity: in a 30-year-old male patient with physiological (50th percentile) distensibility of the ascending $\left(7.5 \times 10^{-3} \mathrm{~mm} \mathrm{Hg}^{-1}\right)$ and descending $\left(5.5 \times 10^{-3} \mathrm{~mm} \mathrm{Hg}^{-1}\right)$ aortas, the expected overall Windkessel volume of the thoracic aorta is
33.2 mL. With impaired distensibility (below the fifth percentile), the Windkessel volume of the thoracic aorta would decrease by approximately one-third to $22.2 \mathrm{~mL}(-33.13 \%)$. This can induce various severe consequences for blood circulation in the cardiovascular system, such as increases in systolic blood pressure and cardiac afterload, diminished diastolic function ${ }^{17}$ and coronary blood flow, ${ }^{18}$ and left ventricular hypertrophy. ${ }^{17}$ High systolic pressure was found to directly result in fractures of the elastic laminae and an increase in collagen fibres, further accelerating the loss of arterial elasticity, ultimately leading to vascular dysfunction, heart failure and cardiovascular events. ${ }^{19}$

Distensibility impairment in our cohort was most clearly observed in patients above the age of 10 years. This supports a concept of aortic distensibility in which the duration a patient is exposed to risk factors can be crucial for the development of distensibility impairment and thus for the likelihood of subsequent cardiovascular events. In line with these findings, increased wall stiffening in patients with CoA and prolonged exposure to abnormal haemodynamics was recently suggested. ${ }^{20}$ However, despite early surgical repair, the distensibility 
of the aorta can remain impaired in CoA, as has been observed in patients with tetralogy of Fallot. ${ }^{21} 22$

A previous study showed that the development of postoperative systemic HTN depends on the duration of preoperative HTN in $\mathrm{CoA}^{23}$ and suggested that the extent of decrease in aortic distensibility is related to the patients' age at surgery. ${ }^{24}$ Possible concepts of the underlying pathophysiology include an ongoing remodelling process of the pressure-sensitive baroreceptors located in the aortic arch, which adapt to the limited contractility of the vessel wall and higher pressure states over time. ${ }^{25}$ Preoperative HTN and a lack of normalisation due to, for example, inadequate antihypertensive treatment after surgical repair may be important causes of subsequent loss of aortic elasticity and thus cardiovascular complications in patients with CoA. In accordance with these concepts, our data show the most impaired distensibility values among patients with uncontrolled HTN despite antihypertensive therapy, whereas well-adjusted blood pressures at the time of the study were associated with improved aortic distensibility of the descending aorta. There is a lack of longitudinal studies regarding the reversibility of distensibility impairment under antihypertensive medication in patients with CoA. However, it has been shown that adequate HTN control may also prevent HTN-related complications, ${ }^{13}$ such as coronary artery disease, sudden cardiac death, heart failure and cerebrovascular accidents. ${ }^{3}$

Patients with BAVD are known to be affected by severe alterations in flow profiles ${ }^{26}$ and degenerative changes in the tunica media of the ascending aorta, which also influence the elasticity of the aorta. ${ }^{727}$ Previous studies have shown increased regional wall shear stress in the ascending aorta of patients with BAVD to correspond with extracellular matrix dysregulation and elastic fibre degeneration. ${ }^{6}$ The bicuspid valve may further contribute by speeding up the progression of aortic wall complications such as aortic dilatation, ${ }^{27}$ aneurysm, rupture and dissection. ${ }^{28}$ Dilated and aneurysmatic areas in BAVD were previously reported to show reduced aortic elasticity. ${ }^{29}$

Our results confirm significantly impaired distensibility of the ascending aorta in patients with CoA and BAVD where aortic dilation was present, starting in childhood. The high prevalence of BAVD and its association with dilatation may superimpose the effects of HTN on the distensibility impairment of the ascending aorta. However, in patients with tricuspid valves, effects of HTN were equally limited to the descending aorta. The high risk for distensibility impairment of the ascending aorta suggests that the presence and severity of BAVD and associated turbulent flow may be important contributors to vascular pathology.

Several techniques have been introduced to assess vascular properties, including invasive intravascular ultrasound, ${ }^{30}$ CT angiography, ${ }^{31}$ echocardiography, pulse wave velocity (PWV) and MRI. ${ }^{32}$ While peripheral PWV measures can be non-invasive and easily applied, they are unsuitable in patients with vascular stenosis, as the narrowing can be a relevant source of error due to abnormal wave reflections. ${ }^{12}$ In contrast to PWV as a current reference standard for assessing arterial stiffness, ${ }^{32}$ the area distensibility used in this study allows localised quantification of elastic capacity, ${ }^{33}$ regardless of the presence of a stenosis. Although the calculation of the area compliance is a relatively new method, its reliability has been previously demonstrated in CoA. ${ }^{12}$

\section{Limitations}

The calculation of area distensibility depends on manual postprocessing of cardiac imaging data, and therefore individual experience as well as training can be essential for precise measurements. To ensure comparability and consistency of aortic compliance and distensibility measurements, the interobserver variability has been assessed. ${ }^{34}$ To reduce the risk of overestimating vessel diameters, angulation errors were reduced using minimal cross-sectional diameters of the vessel. Furthermore, central blood pressure estimations are required for distensibility calculation and were not directly assessed by invasive heart catheterisation. To allow a noninvasive assessment, we used a previously described cuff pressure-based model for central PP estimation ${ }^{33}$ that has been tested in patients with CoA. ${ }^{12}$ All blood pressure measurements were simultaneously obtained to MRI measurements.

The cross-sectional study was not designed to identify a specific age cut-off. The age of 10 years (from which significant associations were found within our model) is only descriptive for our study cohort. Longitudinal studies and larger cohorts are required to assess to which extent these mechanisms apply in principle and why they are more pronounced in adolescents and adults. Moreover, the study was not intended to assess subgroups of heterogeneous previous interventional or surgical techniques.

The comparison of aortic distensibility in CoA with healthy individuals was based on published percentiles by Voges et al. The comparability was ensured by considering the used methods, including sequence parameters as well as data analysis workflow. Published percentiles covered age ranges from 2 to 30 years. As our cohort included older patients, percentile graphs were extrapolated for older patients.

\section{CONCLUSION}

From early adolescence on, BAVD and HTN were both associated with an impairment of aortic distensibility in patients with CoA. Their specific effects differ in strength and localisation: BAVD had its main effect on the distensibility of the ascending aorta, whereas HTN affected the distensibility of the descending aorta. Whether antihypertensive medication is capable of preventing the onset or reversing existing distensibility impairment remains unclear. However, adequate blood pressure control was associated with improved distensibility. Therefore, antihypertensive treatment and the early non-invasive detection of alterations may be pertinent strategies for the 
prevention and reduction of distensibility impairment of the aorta.

\section{Author affiliations}

${ }^{1}$ Charité-Universitätsmedizin, Institute for Computational and Imaging Science in Cardiovascular Medicine, Berlin, Germany

${ }^{2}$ Department of Congenital Heart Disease, Deutsches Herzzentrum Berlin, Berlin, Germany

${ }^{3}$ Centre for Cardiovascular Imaging, UCL Institute of Cardiovascular Science, London, UK

${ }^{4}$ Department of Internal Medicine/Cardiology, Deutsches Herzzentrum, Berlin, Germany

${ }^{5}$ DZHK (German Centre for Cardiovascular Research), Partner Site Berlin, Germany ${ }^{6} \mathrm{BIH}$ (Berlin Institute of Health), Berlin, Germany

Acknowledgements We thank Anne Gale for editorial assistance and Alireza Khasheei for his technical support. MK is a participant in the Charité Digital Clinician Scientist Program funded by DFG.

Contributors TK, VM and MK were responsible for conception and design of the study. MK and AK contributed to subject recruitment. MK and NG acquired imaging data. $N G$ and $L G$ established the measurement method. NG contributed to data acquisition and analysed and interpreted the data. MK and NG conducted the statistical analysis. NG drafted the manuscript. MK and TK critically revised and reviewed the manuscript. SN, JFF, CBL, KR, SR, SS, SK and FB provided feedback on drafts, read and approved the final manuscript. NG wrote the final draft. All authors approved the final manuscript and agree to be accountable for all aspects of the work.

Funding This study was funded by the European Commission under the ICT Program (grant agreement: 611232). LG and MK have received funding in a project supported by the German Research Foundation (DFG, grant 223533535, Berlin, Germany). MK is a participant in the Charite Digital Clinician Scientist Program funded by DFG.

Competing interests None declared.

Patient and public involvement Patients and/or the public were not involved in the design, or conduct, or reporting, or dissemination plans of this research.

Patient consent for publication Not required.

Ethics approval All procedures performed in this study involving human participants were in accordance with the ethical standards of the institutional and national research committee and with the 1964 Helsinki declaration and its later amendments. This study was approved by the local ethics committees (Ethikkommission-Charité-Universitätsmedizin Berlin: EA2/172/13 and National Research Ethics Service Committee London: 15HC23). Written informed consent was obtained from all individual participants and/or their guardians included in the study.

Provenance and peer review Not commissioned; externally peer reviewed.

Data availability statement Data are available upon reasonable request. Please contact the corresponding author to discuss requests for availability of deidentified participant data.

Open access This is an open access article distributed in accordance with the Creative Commons Attribution Non Commercial (CC BY-NC 4.0) license, which permits others to distribute, remix, adapt, build upon this work non-commercially, and license their derivative works on different terms, provided the original work is properly cited, appropriate credit is given, any changes made indicated, and the use is non-commercial. See: http://creativecommons.org/licenses/by-nc/4.0/.

\section{ORCID iD}

Marcus Kelm http://orcid.org/0000-0003-4971-0452

\section{REFERENCES}

1 Canniffe C, Ou P, Walsh K, et al. Hypertension after repair of aortic coarctation--a systematic review. Int J Cardiol 2013;167:2456-61.

2 Toro-Salazar $\mathrm{OH}$, Steinberger J, Thomas W, et al. Long-Term followup of patients after coarctation of the aorta repair. Am J Cardiol 2002;89:541-7.

3 Lee MGY, Allen SL, Kawasaki R, et al. High prevalence of hypertension and end-organ damage late after coarctation repair in normal Arches. Ann Thorac Surg 2015;100:647-53.
4 Tanous D, Benson LN, Horlick EM. Coarctation of the aorta: evaluation and management. Curr Opin Cardiol 2009;24:509-15.

5 Safar ME, Levy BI, Struijker-Boudier H. Current perspectives on arterial stiffness and pulse pressure in hypertension and cardiovascular diseases. Circulation 2003;107:2864-9.

6 Guzzardi DG, Barker AJ, van Ooij P, et al. Valve-Related hemodynamics mediate human bicuspid Aortopathy: insights from wall shear stress mapping. J Am Coll Cardiol 2015;66:892-900.

7 Fedak PWM, Verma S, David TE, et al. Clinical and pathophysiological implications of a bicuspid aortic valve. Circulation 2002;106:900-4.

8 Rose J-L, Lalande A, Bouchot O, et al. Influence of age and sex on aortic distensibility assessed by MRI in healthy subjects. Magn Reson Imaging 2010;28:255-63.

9 Baumgartner H, Bonhoeffer P, De Groot NMS, et al. Esc guidelines for the management of grown-up congenital heart disease (new version 2010). Eur Heart J 2010;31:2915-57.

10 Warnes CA, Williams RG, Bashore TM, et al. ACC/AHA 2008 guidelines for the management of adults with congenital heart disease: a report of the American College of Cardiology/American heart association Task force on practice guidelines (writing Committee to develop guidelines on the management of adults with congenital heart disease). Circulation 2008;118:e714-833.

11 Schäfer M, Morgan GJ, Mitchell MB, et al. Impact of different coarctation therapies on aortic stiffness: phase-contrast MRI study. Int J Cardiovasc Imaging 2018;34:1459-69.

12 Kelm M, Goubergrits L, Fernandes JF, et al. Mri as a tool for noninvasive vascular profiling: a pilot study in patients with aortic coarctation. Expert Rev Med Devices 2016;13:103-12.

13 Mancia G, Fagard R, Narkiewicz K, et al. ESH/ESC guidelines for the management of arterial hypertension: the task force for the management of arterial hypertension of the European Society of hypertension (ESH) and of the European Society of cardiology (ESC) $J$ Hypertens 2013;2013:1281-357.

14 Flynn JT, Kaelber DC, Baker-Smith CM, et al. Clinical practice guideline for screening and management of high blood pressure in children and adolescents. Pediatrics 2017;140:e20171904.

15 Voges I, Jerosch-Herold M, Hedderich J, et al. Normal values of aortic dimensions, distensibility, and pulse wave velocity in children and young adults: a cross-sectional study. J Cardiovasc Magn Reson 2012;14:77.

16 Maldonado J, Pereira T, Polónia J, et al. Arterial stiffness predicts cardiovascular outcome in a low-to-moderate cardiovascular risk population: the EDIVA (Estudo de DIstensibilidade vascular) project. $J$ Hypertens 2011;29:669-75.

17 Eren M, Gorgulu S, Uslu N, et al. Relation between aortic stiffness and left ventricular diastolic function in patients with hypertension, diabetes, or both. Heart 2004;90:37-43.

18 Leung MCH, Meredith IT, Cameron JD. Aortic stiffness affects the coronary blood flow response to percutaneous coronary intervention. Am J Physiol Heart Circ Physiol 2006;290:H624-30.

19 Sun Z, Aging SZ. Aging, arterial stiffness, and hypertension. Hypertension 2015;65:252-6.

20 Juffermans JF, Nederend I, van den Boogaard PJ, et al. The effects of age at correction of aortic coarctation and recurrent obstruction on adolescent patients: MRI evaluation of wall shear stress and pulse wave velocity. Eur Radiol Exp 2019;3:24

21 Schäfer M, Browne LP, Morgan GJ, et al. Reduced proximal aortic compliance and elevated wall shear stress after early repair of tetralogy of Fallot. J Thorac Cardiovasc Surg 2018;156:2239-49.

22 Voges I, Kees J, Jerosch-Herold M, et al. Aortic stiffening and its impact on left atrial volumes and function in patients after successful coarctation repair: a multiparametric cardiovascular magnetic resonance study. J Cardiovasc Magn Reson 2016;18:56.

23 de Divitiis M, Pilla C, Kattenhorn M, et al. Vascular dysfunction after repair of coarctation of the aorta: impact of early surgery. Circulation 2001;104:1165-70.

24 Brili S, Dernellis J, Aggeli C, et al. Aortic elastic properties in patients with repaired coarctation of aorta. Am J Cardiol 1998;82:1140-3.

25 Kenny D, Polson JW, Martin RP, et al. Relationship of aortic pulse wave velocity and baroreceptor reflex sensitivity to blood pressure control in patients with repaired coarctation of the aorta. Am Heart $J$ 2011;162:398-404.

26 Mahadevia R, Barker AJ, Schnell S, et al. Bicuspid aortic cusp fusion morphology alters aortic three-dimensional outflow patterns, wall shear stress, and expression of aortopathy. Circulation 2014;129:673-82.

27 Nistri S, Grande-Allen J, Noale M, et al. Aortic elasticity and size in bicuspid aortic valve syndrome. Eur Heart J 2008;29:472-9. 
28 Keshavarz-Motamed Z, Garcia J, Kadem L. Fluid dynamics of coarctation of the aorta and effect of bicuspid aortic valve. PLoS One 2013;8:e72394

29 Grotenhuis HB, Ottenkamp J, Westenberg JJM, et al. Reduced aortic elasticity and dilatation are associated with aortic regurgitation and left ventricular hypertrophy in nonstenotic bicuspid aortic valve patients. J Am Coll Cardiol 2007:49:1660-5.

30 Stefanadis C, Stratos C, Vlachopoulos C, et al. Pressure-diameter relation of the human aorta. A new method of determination by the application of a special ultrasonic dimension catheter. Circulation 1995;92:2210-9.

31 Ahmadi N, Nabavi V, Hajsadeghi F, et al. Impaired aortic distensibility measured by computed tomography is associated with the severity of coronary artery disease. Int J Cardiovasc Imaging 2011;27:459-69.
32 Cavalcante JL, Lima JAC, Redheuil A, et al. Aortic stiffness: current understanding and future directions. J Am Coll Cardiol 2011;57:1511-22.

33 Saouti N, Marcus JT, Vonk Noordegraaf A, et al. Aortic function quantified: the heart's essential cushion. J Appl Physiol 2012;113:1285-91. (1985).

34 Stoiber L, Ghorbani N, Kelm M, et al. Validation of simple measures of aortic distensibility based on standard 4-chamber cine CMR: a new approach for clinical studies. Clin Res Cardiol 2019. doi:10.1007/s00392-019-01525-8. [Epub ahead of print: $13 \mathrm{Jul}$ 2019] 